\title{
Linguistic and Cultural Analysis of Network Buzzwords in 2017
}

\author{
Xiaojun Liu \\ College of Liberal Arts, Xinxiang University, Xinxiang, 453003, China \\ email: rainbowlxj@163.com
}

Keywords: Network buzzwords; Language features; Cultural connotation

\begin{abstract}
With the rapid development of the Internet, the Internet buzzword has become a unique cultural landscape and has become an indispensable seasoner for people's daily life. In 2017, buzzwords of the Internet have shown diversified and multi-level characteristics. This article selects six 2017 online buzzwords, analyzes their language features and cultural connotations, and probes into the standardization of the use of buzzwords.
\end{abstract}

\section{Introduction}

As a linguistic form, Internet language is a product of the times and the society. With the help of the broad network of communication platform, network language fully reflects people's wisdom and emotions. Network buzzwords reflect the hottest news events of a period or the languages that people use most often at a certain stage. They are the true reflections of people's real-life activities and are also an important part of network culture. This novel way of expression, by the public's favorite, carrying the majority of Internet users express their intentions. [1] Studying and analyzing network buzzwords can make us understand the linguistic phenomena in depth and discover the rules of language at the same time, and we can see through the cultural structure behind the language and promote the standardization of the language. [2]

\section{Language features of network buzzwords in 2017}

Studies in linguistics generally believe that cyber buzzwords are novel, popular, concise, and non-normative. [3] As users of language, we should not only understand but also know why.

\subsection{I may XX Fake X}

One of its origins comes from the E-sports circle. Play CSGO team members like to drink, when not playing well, put the pot rejection to the wine: "I may have drank fake wine", "fake wine harm” ...... Second, from the fighting nation. Russia's unscrupulous traders will personal alcohol detergent products, made of fake wine and sales, lead to poisoning and even death of many people. So, " I may have drank fake wine " on the microblogging spread. Later, some netizens broke the news that when they traveled in 2016, they even saw the fake Terracotta Warriors and Horses in Xi'an. Then, "I might have seen the Fake Terracotta Warriors and Horses" spread again. "

Really let "I may XX Fake X" fire up, is a set of facial expression package during the university final exam: I may have reviewed the fake books, I may have got the fake exam paper, I might have made a false focus, I may have entered the fake exam room, I may listen to a fake class, I may use a false pen, I may be on a fake university, I may have a fake brain, ...... After the enthusiasm of thousands of students forwarded, "I may XX Fake X" to become 2017's first online language. Other examples have followed: I may have had a fake meal, I may have taken a fake medicine, I may have received a fake red envelope, I may have spent fake money, I may have had a false sleep, I may have made a fake boyfriend. ...

"I may XX fake X" has the characteristics of being able to produce strong. "I am XX XX fake X" in the "XX" verb, " $\mathrm{X}$ " with the noun, many words into the structure can be put into immediate use, can produce very strong. After the use of generalization, the meaning of "fake" is no longer "hypocritical, unreal", refers to the speaker's inner feelings, in fact, is an alternative ridicule and 
self-deprecating. [4]

\subsection{Your conscience will not hurt?}

In the first half of 2017, some question and answer website users showed evidence that $\mathrm{Du} F u$ wrote a lot of poems for Li Bai's life. But Li Bai not only did not appreciate, but also wrote a "Gift to Wang Lun" .so many netizens began to laugh: Li Bai, you The conscience will not hurt? In early 2017, "Parrot Brothers" facial expression packs from Japan have become the new web reds. Their special amusing expressions have been added in a variety of texts. So "your conscience won't hurt" is a popular network. The rhetorical question has expressed the helplessness of the extremely injured inner speaker.

\subsection{Heartache, old iron}

When it comes to the source of "Heartache, old iron"I have to mention the abstract fish TV. The audience of this live broadcast studio is basically in the teens to the twenties and thirtysies, their character is publicity, often pop up subtitles. One day, the studio suddenly mixed with a bunch of northeast children, many people made "Heartache, old iron," the phrase barrage, so quickly became popular in the Internet. Which "old iron" is the northeast dialect, which means good friends, but the complete meaning of this sentence is something touched you and caused you more touched or sad emotional factors, known as the "Old Iron ". "Old Iron" comes from dialects, the language has a sense of the image.

\subsection{Pippi shrimp, we go}

The meaning of "Pipi shrimp" on the Internet actually refers to some such as the geoducks, melon skin, and no practical significance. As more and more widely used, Pipi shrimp exists as a virtual, somewhat similar to Jia Junpeng, Xiao Ming, I have a friend ... and so on the same existence, the word originated from a game anchor very much like to say "Pipi shrimp", due to the popularity of this anchor is very popular, resulting in many fans also followed Pipi shrimp. The "XXX we go" originated in the "source Lone Star , we go", it is also a game-related, users will combine the two together, derived from today's new one.

\subsection{Do you have freestyle?}

This is Wu Yifan as a celebrity producer in the IQIYI web variety show "China has hip-hop". When the sea election to some of the strength but did not play a good player plus questions like "Did you have freestyle?", so produced a buzzword, which also extended a lot of expression packages and article use craze.

\subsection{Amazing my XX}

"Amazing my XX" topic volume of 770 million +. There are two versions of the popular "Amazing my XX". Version one: military training, a middle school student playing game, instructors found, and then instructors to help him finish the game, in the process of playing the game, the instructors Super attachment, kill kill, teammates have said: amazing My brother! Used to express a very amazing meaning, so spread out. Version 2: A classmate's gathering, a classmate's finger outside the BMW said, amazing, my teacher. Another classmate pointing to the Mercedes Benz car said: amazing, my doctor. After a classmate Porsche finger said, amazing, my lawyer. I pointed to the taxi outside the door, amazing, my brother.

" Amazing my XX" belongs to the fixed format, in front of the "powerful" is an adjective, such as: powerful my brother; can also fill in the verbs, such as: the promised my brother. Words like this can go into this format. "Amazing my brother", Many express exclamation, surprise emotion color.

The characteristics of network buzzwords in 2017 are as follows: To misinterpret the original meaning, or the new meaning is generated based on the original meaning; mixed Chinese and English; fixed format application; homophonic words, Homophony is popular in Network buzzwords. [5] 


\section{The cultural connotations of the Internet buzzwords in the 2017}

Network buzzwords is a new type of language. As a cultural carrier, Internet buzzwords reflect the cultural demands of the masses of the people and moreover, they experience the cultural emotions of the masses. Network buzzwords reflect all aspects of today's society. The production of buzzwords is closely related to prevalence and cultural life in today's society. [6]

\subsection{The rapid development of network technology}

Network buzzwords are closely related to the development of network technology. The popularity of buzzwords is closely related to the development of information technology. Most of the initial network buzzwords originated in the film and online forums, and then network buzzwords became more and more closely linked with people's social life. The buzzword "I may XX fake X" in 2017, and "Heartache, Old Iron" all reflect the social and cultural life of modern people from different angles.

\subsection{Political and cultural awareness}

The prevalence of online buzzwords is actually a symbol of the people's right to voice. Before the Internet was created, the information was blocked and the public's right to speak hard to operate in the sun. The emergence of the Internet, making people express their views more broadly and freely. [7] The popular phrase in 2017, "Your conscience will not hurt?" Is a manifestation of the masses expressing their own views.

\subsection{Pan-entertainment of contemporary culture}

Network buzzwords humorous, in the expression, people often use the mode of ridicule euphemistically convey the reality. "Pipi shrimp, we go", "you have freestyle it?" The popular Internet users is the way to use ridicule, entertaining buzzwords. [8]

\subsection{The embodiment of the value of contemporary social culture}

The value culture is the correct view and belief generally believed by the masses. In 2017, the popular network buzzword, "Amazing my XX," It reflects our positive attitude towards work and life.

\section{Conclusion}

The network is a double-edged sword. The network buzzwords produced in the network also have two sides. Therefore, we should take a dialectical view. We must not only give full play to its role of enriching language enthusiasm, but also promptly detect and resist the spread of bad language.

In such a highly culturally prosperous era today, cyber buzzwords are infiltrating into our lives with a culture of both sophistication and elegance. We should treat cyber buzzwords with an inclusive mind and then use the media to guide us accurately. You can make the network buzzwords healthy growth. We have to step to lead the network buzzwords into standardization.

\section{References}

[1] Yao Qin. Explore the Language Changes of Internet Catchwords in 2017 from the Angle of Modern Communication[J]. Modern communication, 2017(09):87.

[2] Hui Deng. A Textual Study of the Network Epithet "I May V False N"[J]. Modern Chinese (Language Studies Edition), 2017(04):125-127.

[3] Xiaofeng Zhou, Ge Liu. An Analysis of Popular Words on Internet from the Perspective of Language and Culture[J]. Journal of Northeast Normal University (Philosophy and Social Sciences), 2017(3):184-189. 
[4] Changri Luo, Tingting He. An Analysis of Evolution of Chinese Internet Catchword and Its Effect on Digital Cultural Communition [J]. Journal of Henan University (Social Science), 2015(2): 108-115.

[5] Jingli Huang. A comparative study of the network popular language and the standardization of language and language[J]. Language building, 2016(11):37-38[J].

[6] Yinxia Yu. Pragmatic review of internet popular language[D]. Central University for Nationalities master's degree thesis, 2015.

[7] Ya Dai. Research on Word Variation in Internet Catchwords[D]. Xiangtan University Master's Thesis, 2016.

[8] Kun Yang. The socio pragmatic study of Internet Catchwords[J]. Academic exploration, 2017 (10):84-89. 